\title{
The Impact of Opportunism and Conflict on Non-Economic and Economic Satisfaction in Business Relationships: An Abstract
}

\author{
Janice M. Payan, Carmen Padín, Carlos Ferro, and Göran Svensson
}

\begin{abstract}
Previous studies have investigated long-term buyer-supplier relationships including relational constructs such as noneconomic/economic satisfaction, opportunism, and conflict. However, opportunism is rarely examined in a business marketing setting and rarely studied along with relational constructs such as noneconomic/ economic satisfaction. The construct of conflict also is rarely examined with noneconomic and economic satisfaction. This study examined manufacturer-supplier relationships between Spanish small- and medium-sized enterprises (SMEs). The results of this study show that negative sentiments (opportunism and conflict) in business relationships have a direct negative association with noneconomic satisfaction but only indirectly to economic satisfaction. If opportunism and conflict are not resolved and managed adequately, they will have a negative effect on noneconomic satisfaction and an indirect negative effect on economic satisfaction. It is dangerous to have negative effects (whether directly or indirectly) on both noneconomic and economic satisfaction due to their critical impact on the success of a business relationship.
\end{abstract}

References Available Upon Request

\footnotetext{
J. M. Payan ( $\square)$

University of Northern Colorado, Greeley, CO, USA

e-mail: janice.payan@unco.edu

C. Padín · C. Ferro

University of Vigo, Vigo, Spain

e-mail: padin@uvigo.es; cferro@uvigo.es

G. Svensson

Kristiania University College, Molndal, Norway

e-mail: gosv61@gmail.com
} 